\title{
Improving cooking and eating quality of Xieyou57, an elite indica hybrid rice, by marker-assisted selection of the $W x$ locus
}

\author{
Dahu Ni • Shilu Zhang $\cdot$ Sheng Chen \\ Yong $\mathrm{Xu} \cdot \mathrm{Li} \mathrm{Li} \cdot \mathrm{Hao} \mathrm{Li} \cdot$ Zongyang Wang $\cdot$ \\ Xiuling Cai $\cdot$ Zefu Li $\cdot$ Jianbo Yang
}

Received: 8 December 2010/ Accepted: 29 January 2011/Published online: 15 February 2011

(C) The Author(s) 2011. This article is published with open access at Springerlink.com

\begin{abstract}
Xieyou 57, an elite hybrid rice with high grain yield and broad eco-adaptability, is widely planted in China. Its cooking and eating quality, however, is unsatisfactory due to high-amylose content (AC). In this research, a molecular markerassisted selection method was used to breed for lowamylose content through the modification of the $W x$ genes in both parents of Xieyou 57. The quality of the parent and hybrid lines were then compared before and after modification. Amylose content of GT-type hybrids derived from crosses of Xieqingzao $A(G G) \times$ 057(TT) or Xieqingzao A(TT) $\times 057(\mathrm{GG})$, was reduced to about $19 \%$ from about $26 \%$ in the original hybrid Xieqingzao $\mathrm{A}(\mathrm{GG}) \times 057(\mathrm{GG})$. Wide variation in amylose contents, however, was observed in these GT-type hybrids. With further improvements in both parental lines, the TT type hybrid of Xieqingzao
\end{abstract}

D. Ni · H. Li

Institute of Plasma Physics, Chinese Academy

of Sciences, Hefei 230031, China

D. Ni - S. Zhang $\cdot$ S. Chen $\cdot$ Y. Xu $\cdot$ L. Li $~$

Z. Li · J. Yang $(\bowtie)$

Key Laboratory of Rice Genetics Breeding of Anhui Province/Rice Research Institute, Anhui Academy of Agricultural Sciences, Hefei 230031, China e-mail: yjianbo@263.net

\section{Z. Wang · X. Cai}

Institute of Plant Physiology and Ecology Shanghai Institutes for Biological Sciences, Chinese Academy of Sciences, Shanghai 200032, China
$\mathrm{A}(\mathrm{TT}) \times 057(\mathrm{TT})$ contained even lower amylose $(12.5 \%)$ with good uniformity and exhibited much better cooking and eating quality than the original hybrid of Xieqingzao $\mathrm{A}(\mathrm{GG}) \times 057(\mathrm{GG})$. Meanwhile, yield potential was also improved by increasing panicle size and grain number of the male sterile line. These results demonstrated the success in significantly improving the cooking and eating quality of hybrid rice while maintaining the good agronomical attributes of the parent lines by using molecular marker-assisted breeding in combination with conventional agronomical selection.

Keywords Hybrid rice - Quality ·

Amylose content · Marker-assisted selection . Granule-bound starch synthase .

Rapid viscosity analysis

\section{Introduction}

Since its initial released and promoted in China in 1976, hybrid rice has gained great popularity for its outstanding productivity, wide eco-adaptability and tremendous contributions to China's food security. While the benefit of high yield potential is highly recognized, the poor eating and cooking quality of most rice hybrids poses challenges in meeting consumer expectations. This issue is especially prominent for indica hybrid rice. Therefore, quality improvement has been highlighted as one of the key 
objectives in hybrid rice breeding in order to satisfy the increasing market needs and consumption standards in recent years (Cheng and Li 2007). Breeders and producers start to pay more attention to the composite performance of rice hybrids and varieties in terms of high productivity, good quality, and high efficiency. Meanwhile, the breeding strategies place more emphases on specific improvements of parents using modern molecular methods in addition to the conventional methods that mainly depend on combination of parental genotypes.

Xieyou 57 is an elite indica hybrid developed and released in the beginning of the twenty-first century. It is grown in the mid-lower reaches of the Yangtze river featuring high yield and wide eco-adaptability. However, its poor cooking and eating quality and low seed setting rate in seed production has undermined its acceptance by consumers and the seed industry. The high-amylose content of its parents $(27.3 \%$ in the restorer line 057 and $25.2 \%$ in the male sterile line Xieqingzao A) has been identified as the main cause of the poor quality of Xieyou 57, which makes the cooked rice fluffy, granular, and with unsatisfactory taste. The low yield performance and the associated high production costs in reproduction of the male sterile parent line and in production of hybrid seeds is resulted from the small panicle and low grain number per panicle of the female parent Xieqingzao A. All these obstacles became the major limitations for large scale plantation and adoption of this hybrid (Zhang et al. 2005).

Knowledge accumulated in the past three decades clearly shows that $\mathrm{AC}$ is the key factor affecting rice taste. AC is controlled by the waxy gene $(W x)$ which encodes the granule-bound starch synthase (GBSS). Wang et al. (1990) successfully cloned the $W x$ gene and intensively studied the regulation of its expression. They discovered that a G/T polymorphism at the $5^{\prime}$ leader intron splicing site caused profound changes in the production of mature mRNA for starch synthase and thus affected the AC. If the intron +1 base is a $\mathrm{G}$, the intron would be recognized and then effectively spliced, which results in the accumulation of mature mRNA boosting the GBSS activity to a high level with consequent high AC. However, if the $\mathrm{G}$ mutates to $\mathrm{T}$ at the splicing site, the intron would not be recognized. Therefore, only a cryptic splicing site would be used and the splicing efficiency would be greatly reduced. This causes a low yield of mature
mRNA, low GBSS activity, and considerably low AC (Wang et al. 1990; Cai et al. 1998, 2000). This phenomenon was also confirmed by Frances $\mathrm{H}$ et al. (1998), Hirano et al. (1998) and Isshiki et al. (1998). Cai et al. (1998) developed a CAPS marker (cleaved amplified polymorphic sequence, referred to as PCRAccI) based on the G/T polymorphism and the flanking sequences that were recognized by endonuclease AccI. Using this method, the high-amylose (GG-type) and low-amylose (TT type) cultivars can be precisely distinguished from each other. In this research, we successfully modified the high-amylose trait of Xieyou 57 using this CAPS marker-assisted selection.

\section{Materials and methods}

Plant materials

Rice line 057 , the restorer used in production of hybrid Xieyou 57, was first converted from the GGtype to the TT type at the $W x$ locus. In this conversion 057 was used as the receiver in the initial cross and as the recurrent parent in the following backcrosses. Another restorer line Yanhui 559 of low AC and good quality was used as the donor to 057. Meanwhile Xieqingzao B, the maintainer line of the male sterile line Xieqingzao A, was also converted from GG-type to the TT type at the $W x$ locus. In this conversion, Xieqingzao B was used as the receiver and recurrent parent. The corresponding donor was a maintainer line ND42 with low AC, good quality and large panicle size.

Modification of parental lines 057 and Xieqingzao A

Rice line 057 and the donor Yanhui 559 was crossed, and then backcrossed with 057 as the recurrent parent for four generations. During the backcrosses, agronomic characteristics of selected plants were maintained as similar to 057 as possible except that the low AC trait was maintained by PCR-AccI marker-assisted selection. After self-fertilization of the $\mathrm{BC}_{4} \mathrm{~F}_{1}$ generation, stable lines with low AC and good agronomic traits were obtained. The original restorer line 057 , and the improved line were referred to as $057(\mathrm{GG})$ and 057(TT), respectively. 
Another cross was made between Xieqingzao B and the donor parent ND42. Xieqingzao $B$ was used as the recurrent parent in backcrosses to obtain $\mathrm{BC}_{2} \mathrm{~F}_{1}$ generation. Due to the small panicle and low seed setting phenotype of Xieqingzao B, only two generations of backcrossing were performed in order to retain the big panicle and high grain setting features of the donor parent ND42. During backcrosses, individuals with low AC (selected by PCR-AccI), big panicle and more grains were selected, and other characteristics were maintained as close as possible to that of Xieqingzao B. Finally, elite TT genotype lines with low $\mathrm{AC}$ and better agronomic traits were obtained through self-fertilization. The recurrent parent Xieqingzao $\mathrm{B}$ and the modified line were referred to as $\mathrm{XB}(\mathrm{GG})$ and $\mathrm{XB}(\mathrm{TT})$, respectively. The introgression of the TT waxy region into Xieqingzao A was carried out by crossing Xieqingzao A with selected XB(TT) followed by consecutive backcrossing. The starting Xieqingzao $\mathrm{A}$ and the improved version were designated as $\mathrm{XA}(\mathrm{GG})$ and $\mathrm{XA}(\mathrm{TT})$, respectively.

The $\mathrm{XA}(\mathrm{GG})$ and $\mathrm{XA}(\mathrm{TT})$ were crossed with 057(GG) and 057(TT) to generate four different combinations, XA(GG)/057(GG), XA(GG)/057(TT), $\mathrm{XA}(\mathrm{TT}) / 057(\mathrm{GG})$, and XA(TT)/057(TT). These lines were used in the following research.

PCR-AccI marker analysis and GBSS activity determination

Total DNA was extracted according to the method of Lu and Zheng (1992). The PCR-AccI marker analysis was performed as described by Cai et al. (2002). The forward and reverse primers for PCR were $5^{\prime}$-GCT TCACTTCTCTGCTTGTG- ${ }^{\prime}$ and $5^{\prime}$-ATGATTTAA CGAGAGTTGAA- $3^{\prime}$, respectively. The PCR product is a $460 \mathrm{bp}$ fragment containing the $W x$ gene leader intron splicing site. Upon digestion with AccI restriction enzyme, the amplified PCR product of the GG-type gave rise to two fragments of 403 and $57 \mathrm{bp}$. However, the PCR product of the TT type could not be digested with AccI and only a $460 \mathrm{bp}$ band was revealed after electrophoresis. The heterozygous GT-type showed the 460 and 403 bp bands simultaneously after digestion.

The GBSS activity was determined according to Nakamura et al. (1989). Briefly, fresh developing seeds of plants 10 days after fertilization were collected for crude enzyme extraction and activity determination. Heat-inactivated crude enzyme was used as the control in this analysis. The enzyme activity was expressed as nmol substrate per min pre gram fresh weight.

Evaluation of cooking and eating quality

The AC, gelatinization temperature (valued as alkali spreading, ASV), and gel consistency were determined according to the methods included in the Agricultural Industry Standard NY/T593-2002 issued by the Ministry of Agriculture, P. R. of China. Rapid viscosity analysis (RVA) was carried out using a 3-D rapid viscosity analyzer (Newport Scientific, Australia) according to the recommended procedures of American Association of Cereal Chemists (Reddy et al. 1994).

Assessment of agronomic performance

For agronomic performance assessment of hybrids with and without improvements a randomized block design was used. Two parental lines and four hybrids with three replications each were included in the test. Each plot contained three rows with 11 plants per row. The plant spacing was $14 \mathrm{~cm}$ in the row and $23 \mathrm{~cm}$ between the rows. Observation and recording of rice growth and data processing were performed according to Huang et al. (2006).

\section{Results}

Wx genotype, GBSS activity and AC of the recipient and donator parents

PCR-AccI analysis showed that all the $W x$ loci of the recipients $057(\mathrm{GG}), \mathrm{XB}(\mathrm{GG})$, and their hybrid Xieyou 57 [Xieqingzao $\mathrm{A}(\mathrm{GG}) \times 057(\mathrm{GG})$ ] were GG-type (Fig. 1, Lane1, 2, and 9), while those of the donors Yanhui 559 and ND42 were TT type (Fig. 1, Lane 4 and 5). Details of GBSS activity in the developing seeds 10 days after fertilization and $\mathrm{AC}$ in the mature seeds are shown in Table 1. The GG-type samples consistently exhibited higher AC and stronger GBSS activity than the TT type samples. Thus, the molecular marker faithfully reflected the differences in genotype which determines the difference in enzymatic activity 


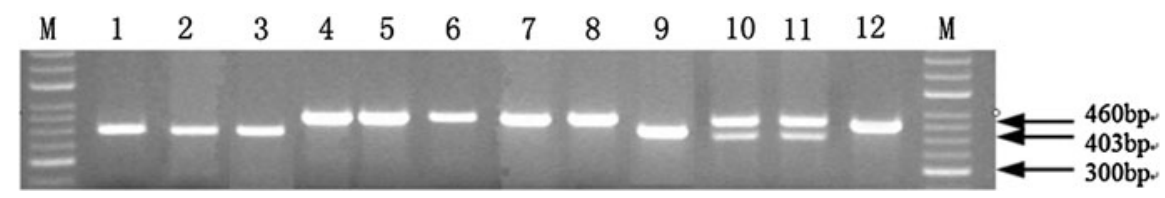

Fig. 1 PCR-Acc I detection of the Wx genotype Lanes 1-10: 057(GG), XB(GG), XA(GG), Yanhui 559, ND42, 057(TT), XB(TT), $\mathrm{XA}(\mathrm{TT}), \mathrm{XA}(\mathrm{GG}) \times 057(\mathrm{GG}), \mathrm{XA}(\mathrm{GG}) \times 057(\mathrm{TT}), \mathrm{XA}(\mathrm{TT}) \times 057(\mathrm{GG}), \mathrm{XA}(\mathrm{TT}) \times 057(\mathrm{TT})$. Lane $M 50$ bp ladder marker

Table $1 W x$ genotype, GBSS activity, and amylose content of parental lines

\begin{tabular}{llllll}
\hline Variety & XB(GG) & 057(GG) & Yanhui 559 & ND42 & Xieyou 57 \\
\hline$W x$ genotype & GG & GG & TT & TT & GG \\
GBSS activity (nmol/g min) & $9.4 \pm 0.25$ & $10.2 \pm 0.65$ & $5.1 \pm 0.14$ & $4.9 \pm 0.52$ & $11.0 \pm 0.52$ \\
Amylose content $(\%)$ & $25.0 \pm 0.31$ & $27.3 \pm 0.32$ & $14.3 \pm 0.29$ & $11.4 \pm 0.23$ & $26.1 \pm 1.53$ \\
\hline
\end{tabular}

Data presented as mean \pm stand deviation

and in the target trait AC. However, the GBSS activity and $\mathrm{AC}$ varied to some degree even in the varieties with same GG or TT genotype (Table 1).

Parent's molecular marker-assisted modification

The recipient and donor lines were crossed and backcrossed following the modification process described in the material and method section. The $F_{1}$ generation which is in a GT heterozygous genotype, was backcrossed separately with their recurrent parents, 057(GG) and XB(GG), and generated two $W x$ genotypes GG and GT. By PCR-AccI marker recognition and selection, the GG-type plants were discarded and the GT-type individuals that had nearly identical phenotype to the recurrent parent were backcrossed consecutively with the recurrent parent. The modification of $057(\mathrm{GG})$ was achieved by one generation of cross, four generations of recurrent backcross, and multiple rounds of selfing. Six stable lines that had the TT genotype and agronomic characteristics similar to the recurrent parent 057(GG) were selected. Of the six lines, ZH171 was most identical to $057(\mathrm{GG})$ and was designated as 057(TT) (Lane 6 in Fig. 1). The modification of $\mathrm{XB}(\mathrm{GG})$ was achieved by one generation of cross, two generations of recurrent backcross, and multiple generations of selfing. Five stable plants were selected based on the TT $W x$ genotype, larger panicle, and the highest similarity of agronomic characteristics to the recurrent parent $\mathrm{XB}(\mathrm{GG})$. Of the five, line nh136 was most identical to $\mathrm{XB}(\mathrm{GG})$ except for panicle size and was designated as $\mathrm{XB}(\mathrm{TT})$ (Lane 7 in Fig. 1). Meanwhile, the TT $W x$ genotype was transferred from $\mathrm{XB}(\mathrm{TT})$ into the isogenic male sterile line $\mathrm{XA}(\mathrm{TT})$ by crossing and recurrent backcrossing with $\mathrm{XA}(\mathrm{GG})$ (Lane 8 in Fig. 1). The AC of the two improved lines, 057 (TT) and $\mathrm{XB}(\mathrm{TT})$, were reduced to 14.3 and $11.9 \%$ from 27.3 to $25.0 \%$, respectively. The GBSS activities dropped to 5.8 and $5.0 \mathrm{nmol} / \mathrm{g} \min$ from 10.2 to $9.4 \mathrm{nmol} / \mathrm{g} \mathrm{min}$, respectively. The AC of $\mathrm{XA}(\mathrm{TT})$ was also reduced from 25.2 to $10.7 \%$ (Table 2).

During the recurrent backcrossing, AC was measured in different lines at the same generation $\left(\mathrm{BC}_{4} \mathrm{~F}_{2}\right)$ of $057(\mathrm{GG})$. Table 3 shows that the $\mathrm{AC}$ value is dependent mainly on the $W x$ genotype as the differences in AC were significant at the 0.01 level between different genotypes despite variations observed among plants with the same $W x$ genotype. These results indicate that in addition to major $W x$ gene, the AC might be affected by other minor modifier genes. Greater variations were observed in GT heterozygous plants.

Cooking and eating quality of the modified and the original hybrids

The comparison of cooking and eating quality before and after modification among parents and their hybrids showed that the hybrid of XA(GG)/057(GG) [marked as XY57(GG)] with GG $W x$ genotype (Lane 
Table 2 Quality measurements of original and improved parental varieties and their hybrids

\begin{tabular}{llll}
\hline Line or hybrid & Amylose content $(\%)$ & Gelatinization temperature (ASV) & Gel consistency (mm) \\
\hline $057(G G)$ & $27.3 \pm 0.32$ & $6.8 \pm 0.09$ & $45.8 \pm 1.12$ \\
$057(\mathrm{TT})$ & $14.3 \pm 0.63^{* *}$ & $5.7 \pm 0.25^{* *}$ & $69.60 \pm 5.79^{* *}$ \\
XB(GG) & $25.0 \pm 0.31$ & $4.2 \pm 0.14$ & $33.3 \pm 2.32$ \\
XB(TT) & $11.9 \pm 0.39^{* *}$ & $4.1 \pm 0.12$ & $87.1 \pm 4.40^{* *}$ \\
XA(GG) & $25.2 \pm 0.52$ & $5.0 \pm 0.18$ & $40.2 \pm 5.20$ \\
XA(TT) & $10.7 \pm 0.34^{* *}$ & $4.0 \pm 0.11^{* *}$ & $74.0 \pm 6.93 * *$ \\
XY57(GG) & $26.1 \pm 1.53 \mathrm{~A}$ & $5.6 \pm 0.44 \mathrm{Aa}$ & $36.5 \pm 2.25 \mathrm{C}$ \\
XY57(GT) & $19.3 \pm 1.72 \mathrm{~B}$ & $5.3 \pm 0.37 \mathrm{Aa}$ & $47.5 \pm 5.53 \mathrm{~B}$ \\
XY57(TG) & $19.2 \pm 2.52 \mathrm{~B}$ & $5.1 \pm 0.24 \mathrm{ABa}$ & $51.5 \pm 7.36 \mathrm{~B}$ \\
XY57(TT) & $12.5 \pm 0.45 \mathrm{C}$ & $4.5 \pm 0.52 \mathrm{Bb}$ & $83.8 \pm 5.71 \mathrm{~A}$ \\
\hline
\end{tabular}

Data presented as mean \pm standard deviation

** Significantly different at 0.01 probability level for comparisons of 057(TT) with 057(GG), XB(TT) with XB(GG) and XA(TT) with $\mathrm{XA}(\mathrm{GG})$, respectively. Data within a column marked by the same upper and lowercase letters indicate no significant difference at 0.01 and 0.05 probability levels for multiple comparisons in all hybrids

Table 3 The $\mathrm{AC}$ values of three $W x$ genotypes in modified progenies $\mathrm{BC}_{4} \mathrm{~F}_{2}$

\begin{tabular}{lccc}
\hline Genotype & Number of plants & AC (mean \pm SD) & AC variation range $(\%)$ \\
\hline GG & 65 & $24.7 \pm 1.97 \mathrm{~A}$ & $20.4-29.4$ \\
GT & 96 & $22.4 \pm 2.41 \mathrm{~B}$ & $17.7-28.5$ \\
TT & 106 & $13.8 \pm 1.43 \mathrm{C}$ & $10.8-17.5$ \\
\hline
\end{tabular}

Data within a column followed by different letters indicate significant difference at 0.01 level

9 in Fig. 1) conferred high GBSS activity $(11.0 \mathrm{nmol} /$ g min), high AC (26.1\%, Table 2), low gel consistency (36.5 mm, Table 2), difficult to cook and cooked rice is fluffy. The two hybrids with a single improved parent, XA(GG)/057(TT) and XA(TT)/ 057(GG), were GT heterozygous genotype and designated as XY57(GT) and XY57(TG) (Lanes 10 and 11, Fig. 1). Their GBSS activities were reduced to 7.03 and $6.33 \mathrm{nmol} / \mathrm{g}$ min, and their $\mathrm{AC}$ values dropped to 19.3 (Table 2) and 19.2\% (Table 2), respectively. Their gel consistencies were softer than original hybrid XY57(GG). These two hybrids had similar $\mathrm{AC}$ values indicating that the $\mathrm{AC}$ was determined mainly by hybrid genotype and that the maternal effect (or cytoplasm effect) was minimal. Therefore, contribution of the parental germplasm with the same $W x$ genotype to the amylose content of hybrids was not significantly different regardless of its role in the crosses, either as male or female parent. However, the amylose content varied remarkably among the hybrid combinations with GT genotype. For example, the AC was $17.2-21.8 \%$ for XY57(GT) and $16.0-22.8 \%$ for XY57(TG) with standard deviations of 1.72 and $2.52 \%$, respectively. This further indicated that the GT or TG heterozygous hybrids had less uniformity in amylose contents. For the hybrids of two modified parents XA(TT)/057(TT), designated as XY57(TT), the GBSS activities and AC were reduced to $4.9 \mathrm{nmol} / \mathrm{g}$ min and $12.5 \%$ (Table 2). Meanwhile, the gel consistency $(83.8 \mathrm{~mm}$, Table 2$)$ was significantly softer than the original hybrid $(36.5 \mathrm{~mm}$, Table 2). The hybrid XY57 (TT) was tender, cohesive, glossy and easy to cook. More interestingly, this TT homozygous genotype had a smaller standard deviation $(0.45 \%)$ and narrower amplitude of variations (11.9-13.0\%) in AC than that of GT and TG heterozygous hybrids, suggesting that less AC uniformity observed in heterozygous hybrids was caused by gene separation. Gelatinization temperature didn't vary correspondingly with the trend of changes in $\mathrm{AC}$ (Table 2). Comparison of the gelatinization temperature among parents and hybrids showed that $W x$ gene had no significant impact on ASV. The AC-determining genes may be independent to genes determining 
the gelatinization temperature. On the other hand, gel consistencies were characterized by coordinated changes with $W x$ genotype and AC values. The trend of gel consistency was TT, GT and GG from high to low. Therefore, introduction of TT genotype into hybrid rice could increase gel consistency and soften cooked rice.

Rapid viscosity analyses of cooked rice provided further evidence for the improvement of cooking and eating quality of the new hybrid XY57(TT). As compared to the original hybrid XY57(GG) and the single parent improved combination XY57(GT) and XY57(TG), the new hybrid XY57(TT) from double improved parents exhibited higher peak viscosity, higher breakdown value, lower recovery value and lower decline value (Table 4), all of which are changes indicative of better cooking and eating quality. Whereas, these quality indicators of the original hybrid were at the opposite end of the spectrum, the heterozygous hybrids XY57(TG) and $\mathrm{XY} 57(\mathrm{GT})$ fell between as expected.

\section{Comparison of the agronomic performance}

In the comparative studies of agronomic traits of the parental varieties and their hybrids, the restorer lines had no significant difference in most traits except the lower tiller number for 057(TT) than 057(GG) (Table 5). The improved XB(TT) showed obvious increases in panicle length and grain number per panicle. At the same time, other traits (e.g. combining ability) were inherited from $\mathrm{XB}(\mathrm{GG})$. For the four hybrids, no significant differences were observed in growth cycle period, plant height, tiller per plant, and seed setting rate. The panicle length and grains per panicle in hybrids XY57(TG) and XY57(TT) were enhanced significantly (difference at 0.05 probability levels). For XY57(TT) from improved male and female parents, although the 1,000-grain weight was slightly reduced, panicle's characteristics and yield per plant were considerably improved. In general, the progenies from double improved parents exhibited significant enhancement in yield and improvement in cooking and eating quality.

\section{Discussion}

Quality trait such as AC is a characteristic endosperm trait having complicated genetic and environmental controlling mechanisms. Although the endosperm is the offspring of a diploid plant, its development and formation is controlled by triploid interactive alleles. The homogeneity and consistency of quality traits in germplasm are profoundly affected by genetic segregation and gene dosage of the major controlling genes. Furthermore, some minor or modifier genes as well as the interactions between genetic and environmental factors exert more influences in the magnitude and quantity of the quality traits. This complexity makes quality trait breeding more difficult and conventional methods less effective. Applying the PCR-AccI molecular marker which is directly linked to the $W x$ gene to the improvement of the amylose content trait, the genotypes can be determined during the whole growth stage and the $\mathrm{AC}$ of

Table 4 Starch viscosity characteristics of original and improved parental varieties and their hybrids [unit: rapid viscosity unit (RVU)]

\begin{tabular}{|c|c|c|c|c|c|c|c|}
\hline Rice line & $\begin{array}{l}\text { Peak } \\
\text { viscosity }\end{array}$ & $\begin{array}{l}\text { Hot broth } \\
\text { viscosity }\end{array}$ & $\begin{array}{l}\text { Breakdown } \\
\text { value }\end{array}$ & $\begin{array}{l}\text { Cold broth } \\
\text { viscosity }\end{array}$ & $\begin{array}{l}\text { Decline } \\
\text { value }\end{array}$ & $\begin{array}{l}\text { Time of } \\
\text { peak }\end{array}$ & Recovery \\
\hline 057(GG) & $2204 b$ & $1787 \mathrm{a}$ & $417 d$ & $3272 a$ & $1068 \mathrm{a}$ & $6.27 \mathrm{a}$ & $1485 \mathrm{a}$ \\
\hline 057(TT) & $2804 a$ & $1602 b$ & $1202 \mathrm{a}$ & $2607 b$ & $-197 \mathrm{e}$ & $6.2 \mathrm{a}$ & $1005 b$ \\
\hline $\mathrm{XB}(\mathrm{GG})$ & $1383 \mathrm{e}$ & $758 d$ & $625 c$ & $1769 d$ & $386 b$ & $5.4 \mathrm{c}$ & $1011 b$ \\
\hline $\mathrm{XB}(\mathrm{TT})$ & $1483 d$ & $1063 \mathrm{c}$ & $420 \mathrm{~d}$ & $1870 \mathrm{c}$ & $387 b$ & $5.93 b$ & $807 d$ \\
\hline XY57(GG) & $2549 b$ & $1845 a$ & $704 d$ & $3457 \mathrm{a}$ & $908 \mathrm{a}$ & $5.93 a$ & $1612 a$ \\
\hline XY57(GT) & $2297 \mathrm{c}$ & $1354 b$ & $943 c$ & $2548 b$ & $251 b$ & $5.6 \mathrm{~b}$ & $1194 b$ \\
\hline XY57(TG) & $2061 d$ & $908 c$ & $1153 b$ & $1589 d$ & $-472 c$ & $5.53 b$ & $681 d$ \\
\hline XY57(TT) & $3384 a$ & $1357 b$ & $2027 \mathrm{a}$ & $2149 \mathrm{c}$ & $-1235 d$ & $5.53 b$ & $792 c$ \\
\hline
\end{tabular}

Data within a column followed by the same lowercase letters indicate no significant difference at 0.05 probability level 
Table 5 Comparison of the agronomic traits of improved parental varieties and their hybrids

\begin{tabular}{lllllllll}
\hline $\begin{array}{l}\text { Line or } \\
\text { hybrid }\end{array}$ & $\begin{array}{l}\text { Days to } \\
\text { harvesting(d) }\end{array}$ & $\begin{array}{l}\text { Plant } \\
\text { height }(\mathrm{cm})\end{array}$ & $\begin{array}{l}\text { Tillers/ } \\
\text { plant }\end{array}$ & $\begin{array}{l}\text { Panicle } \\
\text { length }(\mathrm{cm})\end{array}$ & $\begin{array}{l}\text { Grains/ } \\
\text { panicle }\end{array}$ & $\begin{array}{l}\text { Seed } \\
\text { setting }(\%)\end{array}$ & $\begin{array}{l}\text { Grain weight } \\
(\mathrm{g} / 1000)\end{array}$ & $\begin{array}{l}\text { Yield } \\
(\mathrm{g} / \mathrm{plant})\end{array}$ \\
\hline $057(\mathrm{GG})$ & 139 & 113.6 & 4.7 & 22.4 & 144.3 & 86.1 & 25.6 & 14.3 \\
$057(\mathrm{TT})$ & 140 & 116.0 & 4.0 & 21.7 & 166.2 & 92.2 & $24.6^{*}$ & 15.1 \\
XB(GG) & 118 & 70.0 & 5.3 & 18.5 & 82.9 & 87.0 & 27.1 & 10.0 \\
XB(TT) & 120 & 70.9 & 4.7 & $22.5 *$ & $107.9 *$ & 83.9 & 27.2 & $12.1 *$ \\
XY57(GG) & $143 \mathrm{a}$ & $122.2 \mathrm{a}$ & $5.3 \mathrm{a}$ & $24.0 \mathrm{a}$ & $145.5 \mathrm{a}$ & $92.7 \mathrm{a}$ & $26.9 \mathrm{a}$ & $19.2 \mathrm{a}$ \\
XY57(GT) & $142 \mathrm{a}$ & $122.1 \mathrm{a}$ & $5.8 \mathrm{a}$ & $24.0 \mathrm{a}$ & $133.0 \mathrm{a}$ & $93.7 \mathrm{a}$ & $27.4 \mathrm{a}$ & $21.1 \mathrm{ab}$ \\
XY57(TG) & $143 \mathrm{a}$ & $122.6 \mathrm{a}$ & $4.7 \mathrm{a}$ & $26.4 \mathrm{~b}$ & $198.1 \mathrm{~b}$ & $92.7 \mathrm{a}$ & $26.0 \mathrm{~b}$ & $22.4 \mathrm{ab}$ \\
XY57(TT) & $144 \mathrm{a}$ & $120.4 \mathrm{a}$ & $4.9 \mathrm{a}$ & $27.0 \mathrm{~b}$ & $232.2 \mathrm{~b}$ & $92.6 \mathrm{a}$ & $25.8 \mathrm{~b}$ & $26.7 \mathrm{~b}$ \\
\hline
\end{tabular}

* Significant difference at $5 \%$ probability level between original and improved parent. Data within a column followed by the same lower case letters indicate no significant difference at $5 \%$ probability levels

the progeny can be analyzed accurately. Thus, marker-assisted selection can markedly enhance the efficiency of rice quality trait breeding (Cai et al. 2002; Zhang et al. 2005; Chen et al. 2008).

Using PCR-AccI molecular marker-assisted selection, the restorer line $057(\mathrm{GG})$ and the male sterile line $\mathrm{XA}(\mathrm{GG})$ were improved in amylose content. At the same time, the grain number per panicle of

$\mathrm{XA}(\mathrm{TT})$ increased significantly. AC of the hybrids produced from single improved parent was reduced to intermediate levels. However, a wide divergence in the AC traits was observed in the GT or TG heterozygous hybrids which resulted in wide variation in rice quality. Due to the homozygous nature of $W x$ locus (T/T), XY57(TT) from double improved parents exhibited remarkable improvement not only in the eating and cooking quality but also in the homogeneity among the grains. In addition, the improved XA(TT) with larger panicle and higher grain number per panicle was very useful in enhancing the multiplication of the male sterile line, the production of hybrid seed, and the potential productivity of the hybrid. In this research we not only focused on the key issue of AC but also took the whole quality improvement into consideration as such an integrated technical approach was deployed to combine molecular marker-assisted selection and conventional field selection. Such integrated breeding strategies should have broad applications and prospects in the improvement of crops.

Zhou et al. (2003) had reported that the $W x$ gene determined the $\mathrm{AC}$, gelatinization temperature, and gel consistency of rice. However, Sun et al. (2005) and Liu et al. (2006) found no sensible link between gelatinization temperature and $W x$ genotype. Our results provided further supports to the latter statement. In addition, our results demonstrated that down-regulation of the amylase content caused reduction of 1,000-grain weight, which confirmed the observations by Zhou et al. (2003) and Liu et al. (2006). There might be a QTL (quantitative trait locus) near the $W x$ locus that could be responsible for the phenotype of grain weight variation in the range of 5.2-8.6\% (Yu et al. 1997). The reduction of 1,000grain weight may be affected by this QTL. Meanwhile, from a biochemical perspective, down-regulation of AC synthesis probably resulted in negative effect on the weight of seed because AC was the major component of endosperm. Obviously, more should be learned before a conclusion can be made.

Acknowledgments This research is financially supported by the National Basic Research '973' Program of P. R. China, grant No. 2008CB117003, the National High-tech. '863' Program of P. R. China, grant No. 2009AA101101, and the Talented Youth Foundation, Anhui, P. R. China, grant No. 10040606 Y03.

Open Access This article is distributed under the terms of the Creative Commons Attribution Noncommercial License which permits any noncommercial use, distribution, and reproduction in any medium, provided the original author(s) and source are credited.

\section{References}

Agricultural Industry Standard NY/T593-2002, Quality of food rice, issued by ministry of People's Republic of China 
Cai XL, Wang ZY, Xing YY, Zhang JL, Hong MM (1998) Aberrant splicing of intron 1 leads to the heterogeneous $5^{\prime}$ UTR and decreased expression of waxy gene in rice cultivars of intermediate amylose content. Plant J 14(4): 459-465

Cai XL, Wang ZY, Xing YY, Zhang JL, Hogn MM (2000) Alteration of RNA secondary structure of rice waxy intron 1 caused by naturally ocurred mutations. Acta Photophysiologica Sinica 26(1):59-63

Cai XL, Liu QQ, Tang SZ, Gu MH, Wang ZY (2002) Development of a molecular marker for screening the rice cultivars with intermediate amylose content in Oryza sativa subsp. Indica. J plant physiol mol biol 28(2):137-144

Chen S, Ni DH, Lu XZ, Song FS, Zhang SL, Wang ZY, Cai XL, Li ZF, Wang XF, Li L, Yang JB (2008) Reducing amylose content of hybrid rice Xieyou 57 by molecular marker-assisted selection. Chinese J Rice Sci 22(6): 597-602

Cheng SH and Li J (2007). Chinese modern rice, Jindun publishing house, Beijing, 1,pp 283-324

Frances H, Bligh J, Larkin PD, Roach PS, Jones CA, Fu H, Park WD (1998) Use of alternate splice sites in granulebound starch synthase mRNA from low-amylose rice varieties. Plant Mol Biol 38(3):407-415

Hirano HY, Eiguchi M, Sano Y (1998) A single base change altered the regulation of the waxy gene at post-transcriptional level during the domestication of rice. Mol Biol Evol 15(8):978-987

Huang LZ, Wei XH (2006) Descriptors and data standard for rice (Oryza sativa L.). China agriculture press, Beijing, pp 59-119

Isshiki M, Morino K, Nakajima M, Okagaki RJ, Wessler SR, Izawa T, Shimamoto K (1998) A naturally occurring functional allele of the rice waxy locus has a GT to TT mutation at the $5^{\prime}$ splice site of the first intron. Plant $\mathrm{J}$ 15(1):133-138

Liu QQ, Cai XL, Li QF, Tang SZ, Gong ZY, Yu HX, Yan CJ, Wang ZY, Gu MH (2006) Molecular marker-assisted selection for improving cooking and eating quality in Teqing and its hybrid rice. Acta Acronomica Sinica 32(1):64-69

Lu YJ, Zheng KL (1992) A simple method for isolation of rice DNA. Chinese J Rice Sci 6(1):47-48

Nakamura Y, Yuki K, Park S, Ohya T (1989) Carbohydrate me-tabolism in the developing endosperm of rice grains. Plant Cell Physiol 30:833-839

Reddy KR, Subramanian R, Ali SZ, Bhattacharya KR (1994) Viscoelastic properties of rice flour pastes and their relationship to amylose content and rice quality. Cereal Chem 71:548-552

Sun YY, Lu Y, Dong CL, Wang PG, Huang XQ, Deng XJ (2005) Progress in regulation of rice Wx gene expression. Hereditas 27(6):1013-1019

Wang ZY, Wu ZL, Xing YY, Zheng FG, Guo XL, Zhang WG, Hong MM (1990) Nucleotide sequence of rice waxy gene. Nucl Acids Res 18(19):5898

Yu SB, Li JX, Xu CG, Tan YF, Gao YJ, Li XH, Zhang QF, Saghai Maroof MA (1997) Importance of epistasis as the genetic basis of heterosis in an elite rice hybrid. Proc Natl Acad Sci 94(17):9226-9231

Zhang SL, Ni DH, ChX Yi, Li L, Wang XF, Wang ZY, Yang JB (2005) Reducing amylose content of Indica rice variety 057 by molecular marker-assisted selection. Chinese $\mathrm{J}$ Rice Sci 19(5):467-470

Zhou PH, Tan YF, He YQ, Xu CG, Zhang Q (2003) Simultaneous improvement for four quality traits of Zhenshan 97, an elite parent of hybrid rice, by molecular markerassisted selection. Theor Appl Genet 106:326-331 